\title{
Experimental Research and Realization of Network Virtual Experimental System
}

\author{
Kehao Cao and Fengxia Yang \\ Henan Finance \& Taxation College.Henan.GongYi \\ Corresponding E-mail:11862954@qq.com
}

\begin{abstract}
The virtual experiment is a kind of experimental model based on "virtual reality" technology to create and develop which can have a significant effect on the experimental teaching of computer network, which can not only help students to carry out independent network test and promote student's autonomous learning and learning behavior research, but also can dig the potential ability of the equipment, so as to create a new experimental teaching mode. In this paper, it takes the overview of network virtual experiment as the starting point, based on the interpretation of the connotation of simulation technology, analyzing the requirement of system, so as to discuss the framework of the virtual experiment system based on Lab View.
\end{abstract}

\section{Keywords: Virtual experiment; Computer network; Virtual reality}

\section{Introduction}

With the development of society and the advancement of technology, people had put forward new requirements for computer interaction, and virtual reality technologies are coming out by the demands. It mainly combines computer graphics, multimedia technology, sensor technology, display technology, simulation technology, and networking and other technologies as a whole, which put the construction of virtual scene on the network, so as to enable multiple users on different physical locations to be "immersed" together in a virtual environment, which also can realize the free roaming as well as the communication with other users.

Virtual reality technology is an integrated technology which can make full use of high performance computer hardware and software resources as well as all kinds of advanced sensors. The main technology is the technology of real-time 3D graphics generation, multi sensor interaction and high resolution display technology and so on [1]. The virtual reality technology can generate real-time virtual environment with three-dimensional information, he participants of experiment can have real-time control and interaction like in the real world and the environment [2].

Virtual experiment is a kind of experiment mode which is produced and developed with the development of modern computer technology. Virtual experiment is based on virtual reality technology and virtual instrument technology, in fact, it is the extension of computer science and technology [3].Virtual reality technology can generate real-time, artificial virtual environment with three-dimensional information, the operator (the participant) can enter into, so as to produce realistic feeling, they can have real-time control and interaction like in real world and the environment for. Virtual instrument technology enables the computer to become an all powerful electronic instrument, the use of modern computer graphics environment, it can establish a friendly interface with the virtual instrument panel (i.e. soft panel), the operator can control the operation of instrument through friendly graphical interface and graphical programming language to complete the collection, analysis, judgment, display, storage and data generation [4].

Virtual laboratory can also be called as "Col-Laboratory", which is based on virtual 
reality technology, it is a kind of technology to build virtual experiment environment. The so-called virtual reality technology call also be called as virtual reality technology, which is a rise of new high technology in recent ten years, its definition can be summarized as follows: the virtual reality technology is a kind of technology by using the computer to generate a virtual environment, making the users be "immersed " to the environment through a variety of special equipment, which is a kind of technology to realize the user to be interacted with the natural environment directly. Here, the meaning of "reality" refers to the world of reality or the real environment. Therefore, another name of "virtual reality" is a "virtual environment".Virtual reality is an integrated technology involved in computer simulation technology, image processing and pattern recognition, intelligent interface technology, artificial intelligence technology, sensor technology, speech processing and sound technology, network technology, parallel processing technology and high performance computer system, which is a hot direction in today's computer science research.

\section{The Connotation of Simulation Technology}

The so-called simulation technology is based on the principle of similarity, system technology, information technology, as well as the related technology of the simulation. Taking the computer system, and the application of physical equipment and simulation as tools, using model to study the system of a multi discipline integrated technology. In the era without computer, the simulation carried out using the physical model, which was called as physical simulation, physical simulation can have many advantages, such as vivid,direct, easy to believe, but there is also a lot of limitations,such as the reusing feature is poor, easy to be damaged and so on. Most of the modern simulation technology is based on computer technology [5].Through the computer, we can study the object with mathematical description, modeling programming, and running in the computer. Computer simulation has replaced the traditional simulation model with its advantages, which is the mainstream of the modern computer simulation technology. Therefore, modern simulation technology can also be called as computer simulation.

Computer simulation is mainly digital simulation, it mainly includes three factors, namely, system, system model and computer. The three basic activities that are connected with these elements are the establishment of the model which can be the establishment of the mathematical model that can choose the suitable algorithm and simulation experiments, which can be shown in Figure 1.

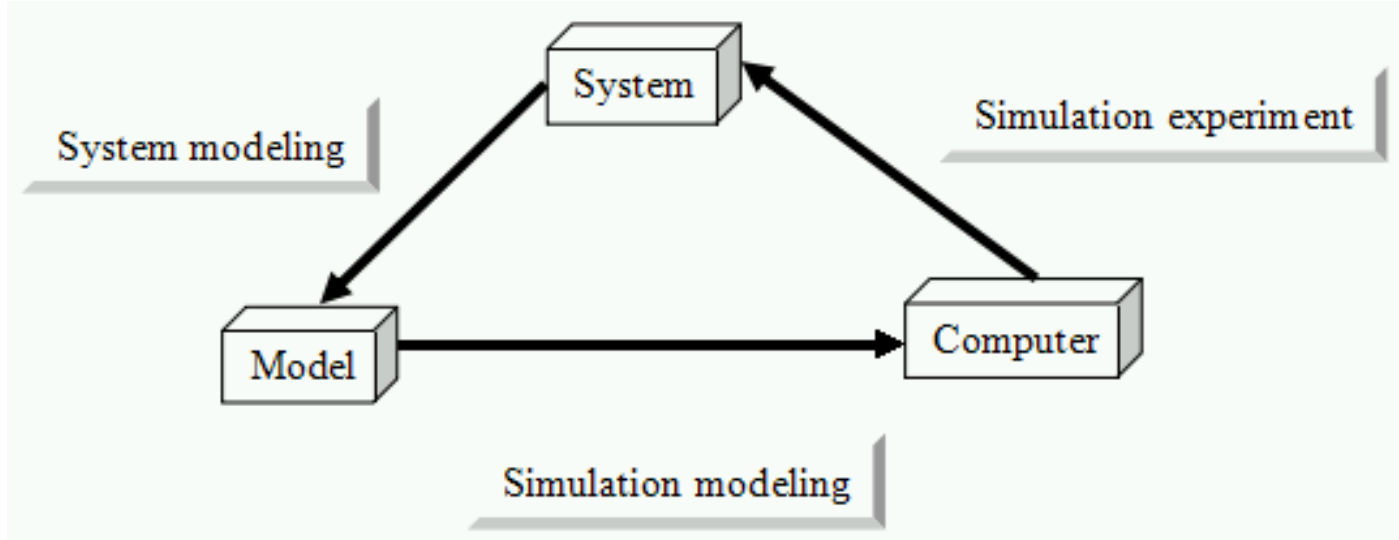

Figure 1. Three Elements and Three Basic Activities of Computer Simulation 


\section{Analysis on the Demand of System}

Computer network virtual experiment system should show the objective things through the real simulation as much as possible, so users can have the feeling of being in the scene, which can improve the interest of learning. System can take the advantages of combining the actual situation, so as to provide virtual simulation equipment, meeting the needs of the experiment, which can solve the problem of the actual conditions of the network that does not have the hardware or network environment. Computer network virtual experiment system simulation can adopt various types of switches and routers, using the Windows platform and Linux platform with PC. Users can drag and drop to the experimental platform by using a variety of devices, which can be composed of network topology and carry on with test. The typical experiments of system simulation can be including Linux file management, Linux common command, Linux process, VI editor and user management experiment; the basic network under Linux and Windows environment, resource sharing, firewall, virtual private network (VPN), DNS device, HTTP service, FTP service, DHCP device, as well as Samba Device, while the experimental routers can be including statistic router device, dynamic router device, RIP protocol, IGRP protocol, OSPF protocol and ARP exchanging machine, VLAN device and VTP experimental device and son on, which can be more than ten types of typical experiments.

\section{Virtual Experiment System Based on View Lab}

View Lab can be used in the following aspects, namely, data acquisition and control, data analysis and data expression. It can provide a new method of programming, which can be called as "virtual instruments"(VIS). It can use virtual instruments software to have the combination operation with image so as to consist the virtual experimental system.

Lab View is a programming development environment, compared with the other computer language, Lab View can use graphical programming language $G$ for programming, the generated program is in the form of block diagram; Lab View is also a general programming system, with a huge function library to complete any programming tasks. The function library of Lab View can be including data acquisition, GPBI, serial control, data analysis, data display and data storage and so on. Lab View can also have the traditional program debugging tools, such as setting breakpoints, displaying data in the form of animation, getting the results of the program (sub VI), single step execution and so on, which can be easy for the adjustment of program.

\section{The Advantage of RIA}

RIA can comply with the developing trend of network applications, such as, cross platform, mobile communication and supporting industry standards (such as XML and Web Services). It also has the advantages of the network, namely: cross platform; instant configuration; RIA can provide the operating environment similar to desktop software, real-time reflection, interactive operation (such as the operation of drag and drop); finally, it can combine the multimedia display, which even provide real-time chatting, voice or video communication and other functions. RIA can combine a variety of advantages, which can be shown in Figure 2. 


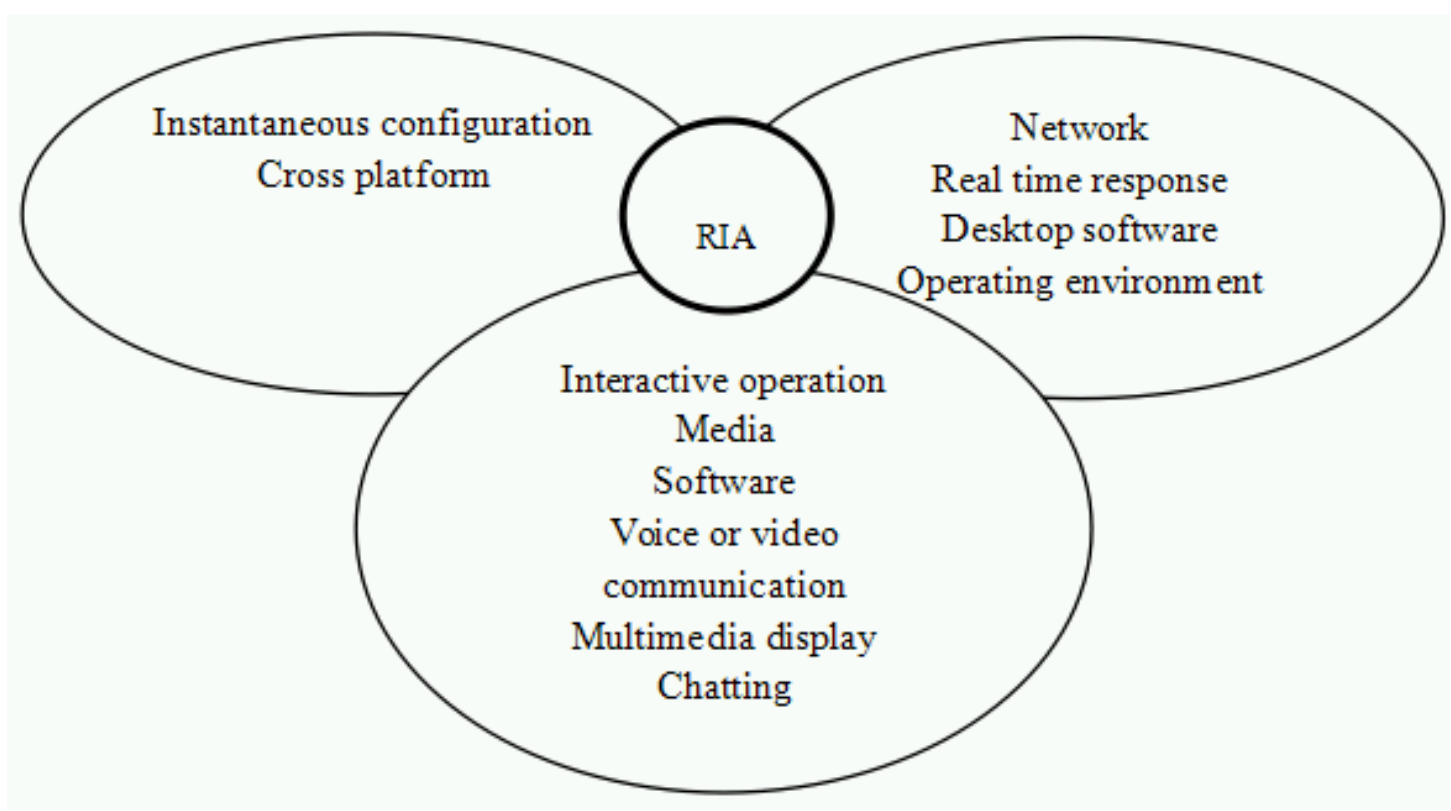

Figure 2. The Advantage of RIA

The Principle of Virtual Experiment System Based on Lab View

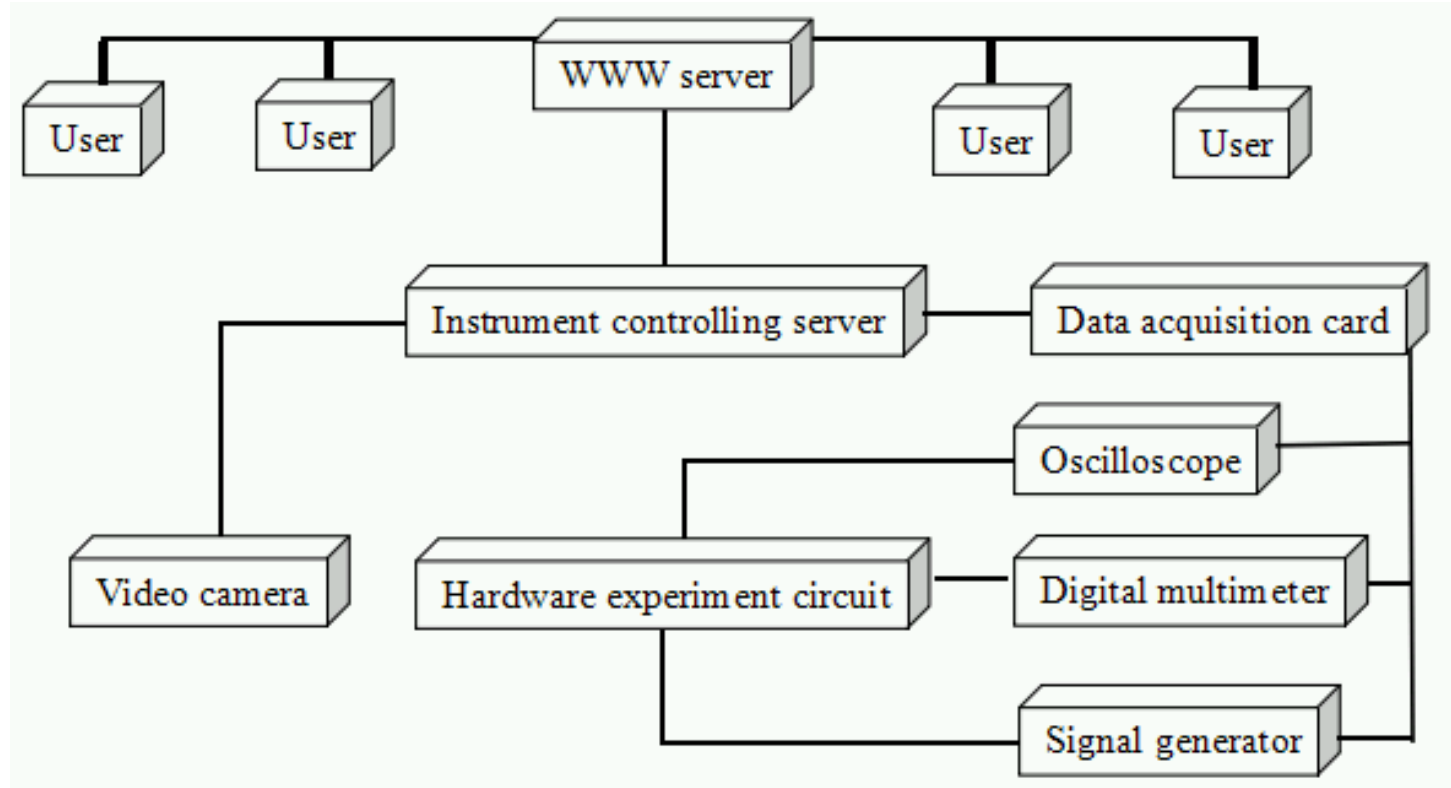

Figure 3. Diagram of Hardware of Remote Virtual Laboratory System

In Figure 3, the computer in the remote virtual lab can connect with all kinds of practical instruments through data acquisition card interface and the connecting lines, such as oscilloscope, function generator, digital data and so on. When the remote users log to the lab through Internet network, they can control the operation over these equipment.

WWW server can generate pages dynamically, the used technology can be CGI, JAVA or ASP and so on. It will generate a virtual experiment system interface based on the current state of the experimental hardware (acquired by the instrument control service) to get a virtual experiment system interface. The user can change the state of the instrument in the virtual experiment interface through various inputting devices, and data can be 
submitted to WWW server, at the same time, WWW server can call the instrument control service to carry on with data processing, which can transfer it into a real test system to accept the change of the real experimental system.

The instrument control service is based on the Lab View platform, its rich device driving support and powerful library function can make the control over the instrument more flexible. It can use thousands of devices for data acquisition, these devices can be including: GPIB, VXI, serial port equipment, PCL, as well as plug-in data acquisition card and so on. It can obtain data through the network, interactive communication and structured query language (SQL) and other ways with the data sources.

Lab View contains a rich content of advanced analysis library, which can carry on the signal processing, statistics, curve fitting and complex analysis. After the data being collected, the experimental staff can use the powerful data analysis program of Lab View to convert the raw data into meaningful results.

\section{The Related Concept of Virtual Machine}

The so-called virtual machine is a virtual computer, there are many types of virtual machines, the virtual machine commonly adopt virtual machine software to complete the implement.Virtual machine software is located between the operating system and hardware, which allows multiple operating systems to run simultaneously on the same host. Physical computer system actually exist in the virtual machine that can be called as host machine, which can also be regarded as the host computer; the logic host computer that use virtual machine software can be called as the virtual machine, which also can be called as client computer. The system that is installed on the host operating system can be called as the host operating system; the system that is installed on the virtual machine operating system can be called as virtual machine operating system. The step of building a virtual machine system method can be as follows: firstly, it can install the VMware virtual machine software in the host operating system (HostOS); then, it can build a new virtual machine in VMware and provide its support for the virtual machine operating system. However, the virtual machine can take up more host memory, in order to ensure the smooth operation of virtual system, thus, it is proposed that the host computer should have a higher configuration for the hardware.

\section{Virtualization Technology}

Before the emergence of virtualization technology, the operating system running is on top of the hardware, the application process can acquire and obtain the right to use hardware by means of interruption, these hardware can be including controllers, memory, hard drives and network card and so on. The dispatching work of hardware cab be dealt by the operating system, when multiple servers provide the service together, some of servers may be in the idle situation, while others are in busy situation, but the utilization rate can even be reached. This unbalanced usage of resources and uneven situation can be encountered by traditional physical machine easily, which is also relatively difficult to be solved.

After the emergence of virtualization technology, there is a change in the physical machine model, which can be shown in Figure 4. The host operating system is no longer in charge of management hardware,but it adds a layer in the middle of the virtual machine monitor, which is responsible for scheduling the use of the hardware. The disadvantage of this model is able to overcome the above-mentioned conventional physical machine so that the hardware utilization can be maximized and improved. The virtualization technology is the resource of computer's hardware, such as memory, network, and storage that are assigned with abstract meaning and converted to the client operating system that can enable the client operating system adjust these resources better than the traditional way.These virtual resources are not restricted by the current architecture, geography or 
physical state of the hardware resource. It can expand the capacity of the hardware, moreover, the software re-configuration process can be simplified. Virtualization can be used to simulate multiple concurrent single stimulation, allowing a platform to exist a number of different operating systems at the same time, and the application program can run independently within their respective spaces without affecting each other, thus it can increase the utilization and efficiency of the physical machine significantly.
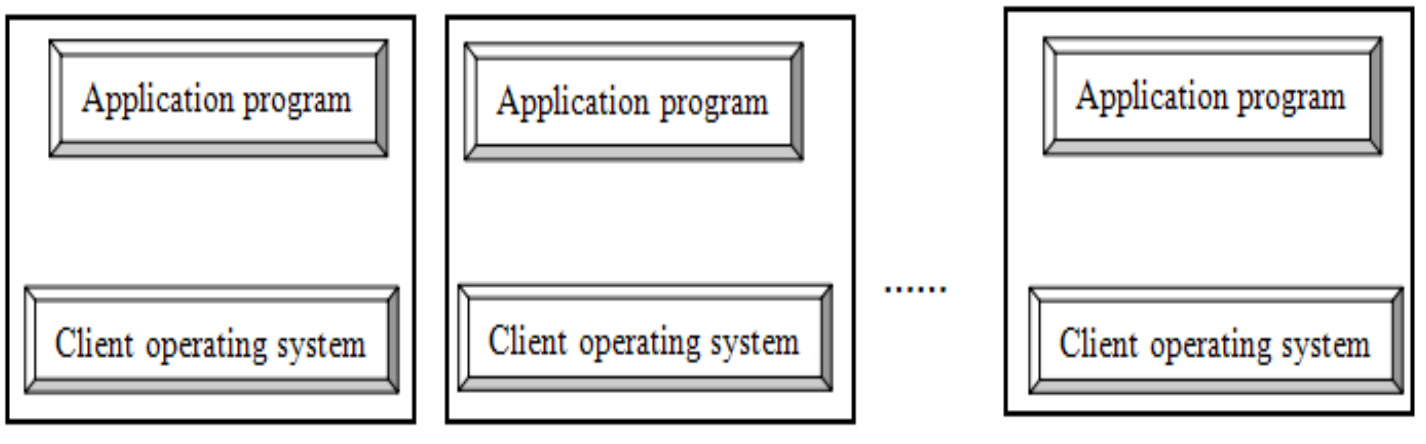

Monitor if virtual machine

\section{Hardware}

Figure 4. The Architecture Virtualization System

We can land router 1 and router 2 , respectively, by using routing and remote accessing service, it can achieve the soft route, linking the two neighboring subnets, allowing the neighboring computers to communicate with each other in the two adjacent subnets. Testing the connection between $\mathrm{pc} 1$ and $\mathrm{pc} 2$, it will find out that the connection fails. From the diagram of network topology we can see, router1 is connected with the subnets 192.168.80.0 and 192.168.81.0, router2 is connected with the subnets 192.168.81.0 and 192.168.82.0. Therefore, in order to achieve three sub-network connection, as long as it can add the 192.168.82.0 static routes on the subnet's router1, and add 192.168.80.0 static route on the subnet's router2. Multiple IP subnets with static routes can use VMware to build complex network. Meanwhile, the the routing and remote access can be set with the static routing function in the virtual machine, so that among these three subnets, the virtual machines can communicate with each other, which can be shown in Figure 5. To build the network, it needs to build three virtual switches and four virtual machines, the network can be set as follows: 


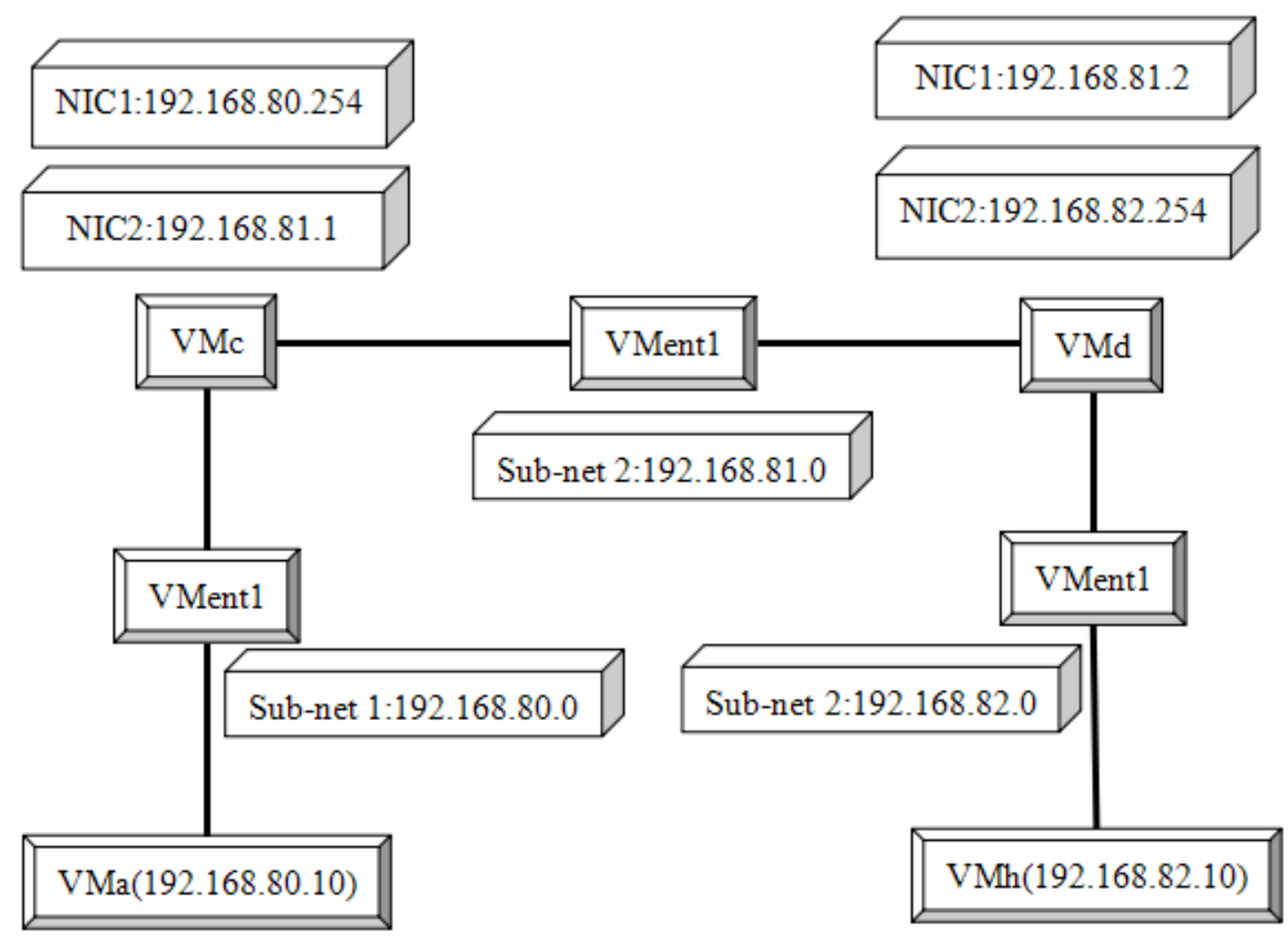

Figure 5. VMware Set Virtual Network with Multiple Subnets

\section{Attachment Strategy}

As for the attachment strategy, "8255 paralleled to interface network virtual experiment" is mainly to identify and implement the connection strategy, and the main code can be seen as follows:

1) Definition of variable

final public static int Zero_Selected $=0$ :

final public static int one_Selected=1:

public static int Connect Status=Zero_Selected:

Public static J Port[ ]p:

The first two lines defined two constants, the latter two lines defined the status variables, namely, Connect state and array of J Port.

2) The initialization of system: the initialization related to policy is in the init function of VirtualLab class.

$\mathrm{P}=$ new J Port[2]; / / initialization the number of J Port array

Buffer ConPolicies(): / / buffer connection strategy

3) The process of policy identification: the process is triggered when users click on the virtual component pin.

When user clicks on the virtual device pin, mouse Clicked function can be executed: when the value of Connected status is Zero_Connected, the program will store the name as well as the object into the first element of the two element global array, changing the Connect status into One_selected; when the value of Connect status is One_Selected, the program will change the state to Zero_Selected, which also can store the pin's name and 
the object in another element, after that, the Test Connect function can be activated.

4) Policy implementation process: this process is performed by the the object of virtuallab, namely, Test Connect function.

\title{
Conclusion
}

The computer interface technology of network virtual experiment system can use the method of component technology, virtual reality technology, combined with database technology, it can use JAVA programming language to write the simulation control platform to construct a virtual electronic laboratory, therefore, students can carry out a series of computer interface technology experiments. Virtual computer interface technology laboratory should have strong interactive function and simulation environment for experiment, which is in accordance with the requirements of remote education to the experimental teaching. It can help students to understand the experimental principle and the related experimental knowledge, it also can create a realistic experimental environment with powerful interactive function, which can make the experiment achieve real results.

\section{References}

[1] Weiss P.L, Jessel A.S. 1999. Virtual reality applications to work. Occupational Health and Industrial Medicine, vol.40, pp:1.

[2] Nomura Junji, Sawada Kazuya. 1999. Virtual reality technology and its industrial applications. Control Engineering Practice, vol.7, pp:1381-1394.

[3] Marinov Valery, Andonov Ivan. 1997. Virtual experiment as an educational aid in tribology of cutting. Computer Methods in Applied Mechanics and Engineering, vol.147, pp:323-327.

[4] Mills Stella, Madalena T de Araújo, Maria. 1999. Learning through virtual reality: a preliminary investigation. Interacting with Computers, vol.11, pp:453-462.

[5] Dias JMS. 1997. Multiuser 3D Virtual Environment. Computer Graphics and Applications, vol.17, pp:24-28.

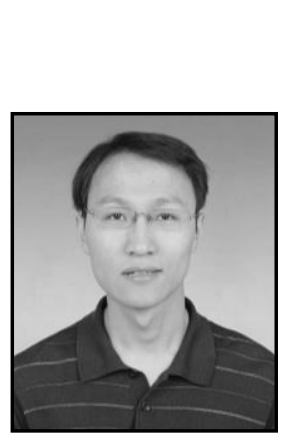

\author{
Authors \\ Kehao Cao, He is a lecturer Research direction: Virtual \\ Experimental System .Data mining
}

Fengxia Yang, assistant professor Research direction: Virtual Experimental System .Data mining 